\title{
Condition detection of transformer winding based on entropy weight correlation theory
}

\author{
Guochao Qian ${ }^{1,}$, Huaitong Yin ${ }^{2}$ and Dexu Zou ${ }^{1}$ \\ ${ }^{1}$ Power Test and Research Institute, Yunnan Municipal Electric Power Company, Kunming, Yunnan Province \\ ${ }^{2}$ Wenshan Electric Power Supply Bureau. Yunnan Power Grid Corporation, Ltd
}

\begin{abstract}
To effectively detect the hidden fault of transformer winding, the combination of grey correlation analysis and entropy weight theory is proposed to detect the winding condition of power transformer based on the inherent relations of vibration frequency response (VFR) curves of several measured points. The vibration frequency response experiment is made on a real $220 \mathrm{kV}$ transformer winding both in normal and loosened conditions. The grey correlation degree and grey area correlation degree of VFR curves in each measured point. Then the entropy weight correlation degree is defined to determine the weight value of all the obtained features. The results have shown that the detection results based on the proposed method are agreed well with the preset condition of transformer winding, which is better illustrated the loosened degree of transformer winding. Compared with the existed FRA method, the vibration frequency response method is more sensitive to the variations of winding condition and has high application value.
\end{abstract}

Keywords: transformer; winding; vibration frequency response; entropy weight correlation; condition detection.

\section{Introduction}

According to statistics show that most of the transformer fault from the outlet at the short circuit. Under the action of strong short circuit impulse current, the winding of the transformer can be easily deformed or loosened [1]. At the same time, the deformation of transformer winding has a cumulative effect. Long term operation of the transformer with hidden troubles may cause the transformer winding insulation breakdown and so on. Even in the short circuit shock, such as the collapse of the transformer windings and other failures, endanger personnel safety and bring huge economic losses. Therefore, it is necessary to study the accurate and efficient method to detect the transformer winding condition, to ensure the safe and reliable operation of the power transformer.

In recent years, due to its high sensitivity and easy to realize online monitoring and other advantages, the vibration detection method of transformer winding, which is based on the dynamic characteristics of windings, has been widely concerned by researchers at home and abroad. It has become one of the important research directions in the field of transformer condition monitoring. In [2-4], the researchers from the theoretical modelling of the vibration characteristics of the transformer windings are studied, and got some meaningful results.; In [5-6], the researchers have studied the

a Corresponding author : 41037504@qq.com

(C) 2016. The authors - Published by Atlantis Press 
development and application of the transformer vibration monitoring system, and got some meaningful results; In [7-8], the researchers have studied the method of detecting the state of the transformer winding based on the vibration signal, and got some meaningful results. Among them, in [9-10], the Spanish scholars put forward according to the transformer vibration model of the calculated results and the measured results are compared to the change of the threshold of the winding state detection. In [11], based on the load voltage and load current as the input variables of the transformer tank vibration model, to predict the transformer winding fault. $\operatorname{In}[12]$, according to the main spectral components of the vibration signal of the transformer, a fundamental frequency conversion model is proposed to determine the fault types of the transformer windings and identify the faults of the windings. However, there are many kinds of transformers, which are closely related to the structure of the vibration signal, the structure of the transformer, the position of the measuring point and the running environment. The validity of the proposed criterion is still to be verified. In [13-14], the difference of Vibration Frequency Response and Frequency Response Analysis in transformer winding deformation detection is analysed and compared. Describes the application of VFR in transformer windings status detection, however, VFR curve of test characteristics description is relatively simple, and do not consider multiple correlation of measured point between VFR curve, still need further study.

Taking into account that the transformer winding is loose, the short circuit impedance, distributed capacitance and distributed inductance are limited. In this paper, based on the vibration frequency sweep test of a certain $220 \mathrm{kV}$ transformer winding under normal and loose. Based on the correlation between the VFR curve at each measuring point of the transformer tank wall and the area correlation theory, the entropy weight correlation analysis method is proposed to detect the state of the transformer winding. At the same time, the test results of FRA are given.

\section{Test system description}

\subsection{Testing principle}

The vibration of transformer in normal operation mainly comes from the vibration of the iron core and the winding. The winding vibration is mainly caused by the electric power generated by the interaction between the winding current and the magnetic leakage field, which can be obtained by the test of the transformer short circuit test. In this paper, the axial vibration characteristics of transformer windings are described in this paper.

The formula (1) can be expressed as the axial vibration acceleration of the windings according to the transformer winding spring mass equivalent model [14].

$$
a=-A \omega_{0}^{2} e^{-\frac{C t}{2 M}} \sin \left(\omega_{0} t+\theta\right)-4 \omega^{2} G \sin (2 \omega t+\psi)
$$

Obviously, the following conclusions can be made by the formula (1).

(1) The axial vibration of the transformer winding mainly include the sine attenuation component of the natural frequency of the winding and the sinusoidal steady state component with the excitation current two harmonic.

(2) The vibration response of the transformer winding is closely related to its natural frequency, stiffness, damping ratio and excitation force.

(3) When the frequency of the excitation force is close to or equal to the natural frequency of the transformer winding, the vibration response of the transformer winding near the frequency will increase obviously.

(4) When the transformer winding appears loose or deformation, the vibration response characteristics will be changed.

Therefore, the excitation current with controllable amplitude and variable frequency is injected into the transformer winding. By placing the vibration sensor placed on the wall of the transformer 
box, the winding vibration signal can be obtained, then the VFR curve of the transformer winding can be calculated. Taking into account the main frequency spectrum of the vibration signal of the transformer winding is usually two times of the excitation force. In this paper, according to the change of the two harmonic VFR curve of the transformer winding, the winding condition is detected.

\subsection{System composition}

The transformer winding vibration frequency sweep test system consists of power supply, step-up transformer and vibration analysis system is composed of three parts. It is as shown in Fig. 1. In Fig.1, the frequency sweep power output is constant and the frequency is variable, and the main output parameters are shown in Table 1. Among them, the amplitude and frequency range of the current is considered as the natural frequency of the transformer winding, the signal to noise ratio of the vibration signal and the portability of the field test. Vibration acquisition and analysis system comprises a data acquisition part, a control module and a display terminal. The constant current control of the output current amplitude of the sweep frequency power supply is realized by the signal of the vibration signal of the wall of the transformer and the current signal of the injection winding.

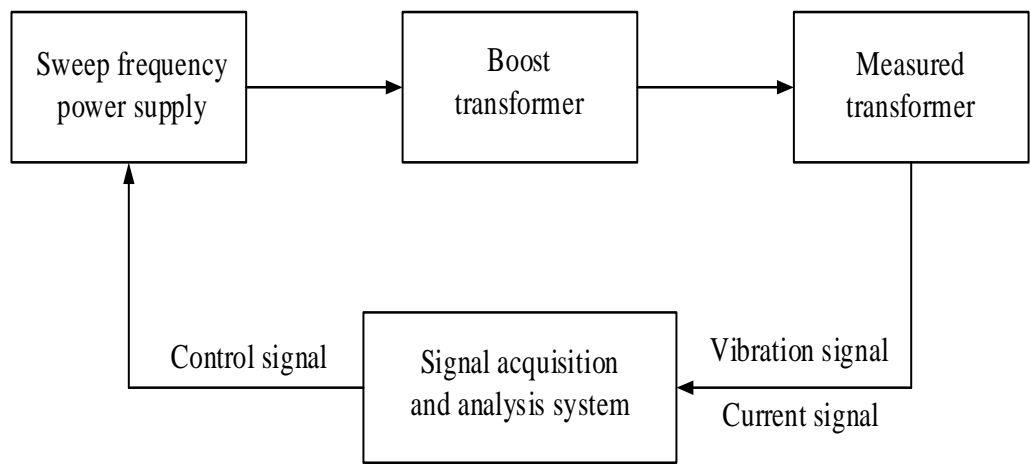

Figure 1. Schematic diagram of vibration frequency response measurement system of transformer winding

Table 1. Main parameters of vibration sweep frequency power supply

\begin{tabular}{|c|c|c|}
\hline $\begin{array}{c}\text { Output } \\
\text { current/A }\end{array}$ & Output current/Hz & $\begin{array}{c}\text { Total harmonic } \\
\text { distortion \% }\end{array}$ \\
\hline $0-10$ & $45-310$ & $\leq 2 \%$ \\
\hline
\end{tabular}

\section{Sweep frequency test of transformer winding}

Figures and tables, as originals of good quality and well contrasted, are to be in their final form, ready for reproduction, pasted in the appropriate place in the text. Try to ensure that the size of the text in your figures is approximately the same size as the main text (10 point). Try to ensure that lines are no thinner than 0.25 point.

\subsection{Test description}

Using self-made vibration frequency response test system on a model for SFP7-120000/220 of power transformer winding sweep frequency test. During the test, the test transformer low voltage side short circuit, high voltage side injection constant current frequency conversion current. At this time, the transformer voltage is low, the core vibration can be neglected, the vibration of the transformer tank wall is mainly due to the winding vibration. Among them, the sweep frequency power output current is $8 \mathrm{~A}$, sweep frequency range is $45 \mathrm{~Hz}-310 \mathrm{~Hz}$, the frequency interval is $1 \mathrm{~Hz}$. 
In addition, taking into account the complexity of the wall structure of the transformer and the influence of different vibration sensors on the test results of vibration signal. According to field test conditions, measured in test transformer box wall 11 vibration sensor, as shown in figure 2.

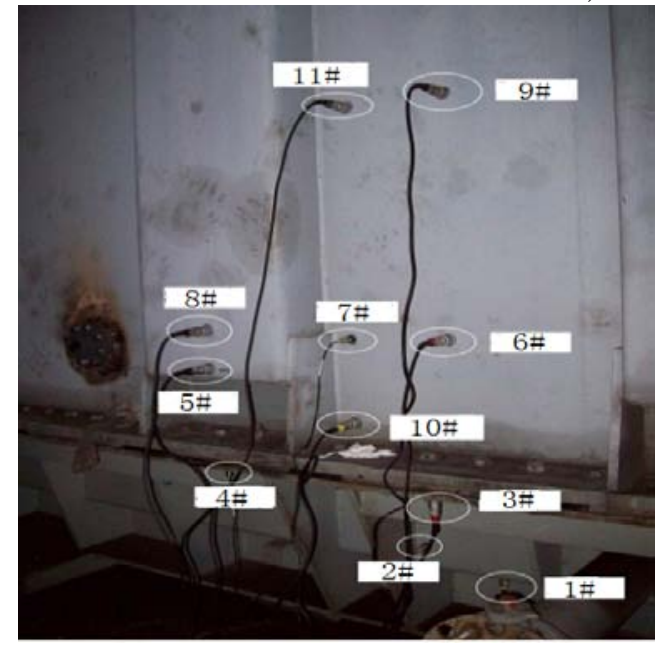

Figure 2. The placement of vibration sensors

The degree of loosening of the transformer winding is simulated by adjusting the A phase winding, as shown in figure 3.Loosening conditions are set respectively: the pressure screw loose $20 \%$, record as loose 1; press the screw loose $40 \%$, record as loose 2; pressure screw loose $60 \%$, record as loose 3.

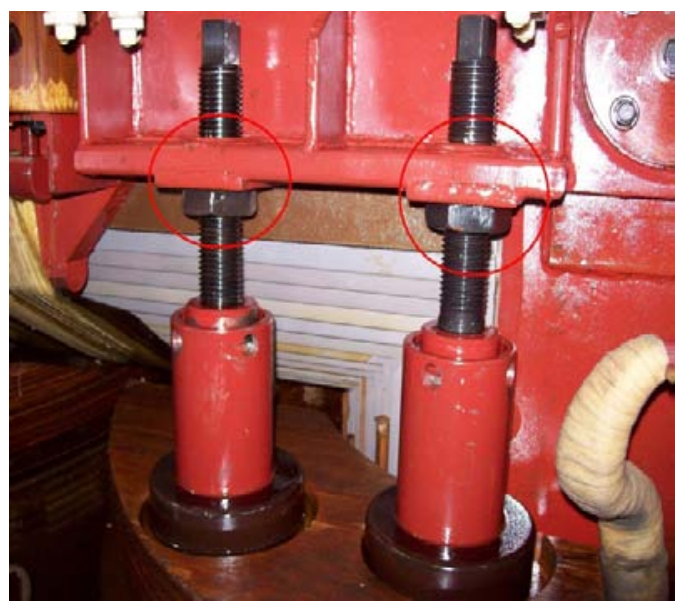

Figure 3. Preset of winding looseness of transformer winding

\subsection{The results of transformer winding sweep frequency}

You are free to use colour illustrations for the online version of the proceedings but any print version will be printed in black and white unless special arrangements have been made with the conference organiser. Please check whether or not this is the case. If the print version will be black and white only, you should check your figure captions carefully and remove any reference to colour in the illustration and text. In addition, some colour figures will degrade or suffer loss of information when converted to black and white, and this should be taken into account when preparing them.

In this paper, the VFR curve of the point 6 and point 7 of the transformer winding under normal working condition is given, as shown in Figure 4. The two sets of curves were obtained by the two 
sweep frequency test, and the test time interval was one day. The purpose of the test is to verify the repeatability of the test. The VFR curves of the same test point in the two sweep frequency test are in good agreement, as shown in Figure 4.The general trend of VFR curve at each test point is the increasing trend with the increase of frequency. And there is an obvious peak point. Due to the influence of the vibration transfer path, the VFR curves at different measuring points are in different shapes.
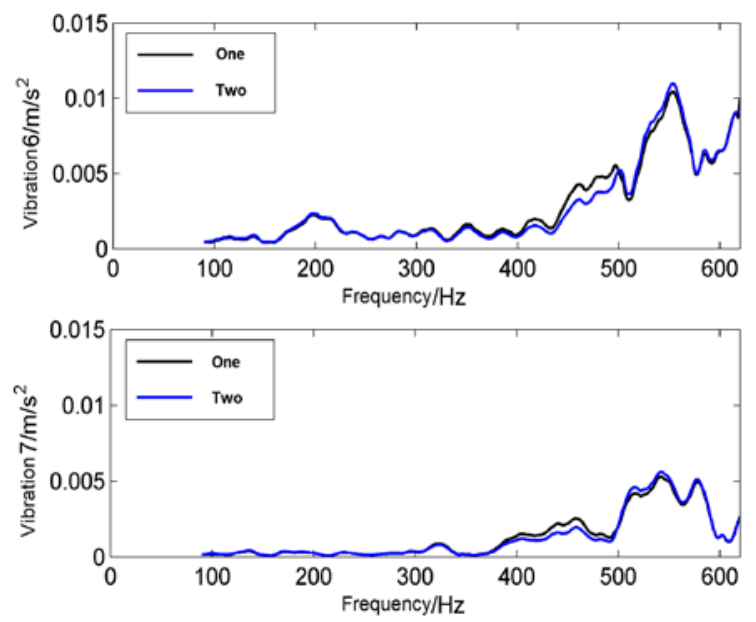

Figure 4. VFR curves under normal condition
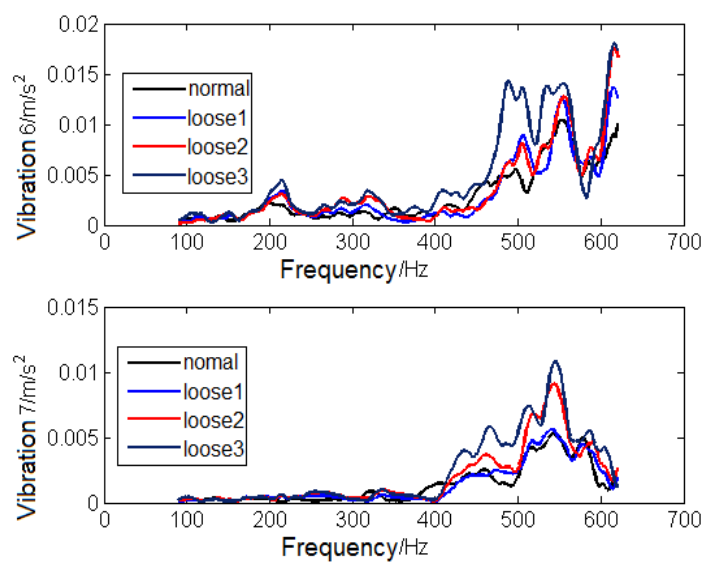

Figure 5. VFR curves of under normal and fault conditions

The VFR curve of the test point 6 and point 7 under the condition of the normal winding and the loose condition of different windings is shown in Figure 5. When the transformer winding is loose, the VFR curve of each test point has a significant change and varies with the measuring point, as shown in figure 5. The specific performance is as follows: the vibration amplitude of some frequency bands is increased, and the peak value of vibration in some frequency bands appears. The reason is that the transformer winding is loosened, the vibration amplitude will increase with the decrease of preload. In addition, the winding loose will make its natural frequency change, and the change of the natural frequency will lead to the peak of the VFR curve shift. 


\section{Entropy weight correlation theory}

The test results show that the VFR curve at each test point of the transformer winding is different but there is a certain correlation. In order to seek the quantitative index of the change of the VFR curve of the transformer winding, an accurate and comprehensive description of the state of the transformer winding is achieved. In this paper, the correlation between the VFR curves of the same measuring point is measured by the grey correlation degree and the area correlation degree. The geometry of the VFR curves and the surrounding area are described respectively, and then the entropy weight correlation degree of the VFR curve at each measurement point is defined to detect the state of the winding. In [15], the biggest advantage of the gray correlation analysis method is that it can effectively deal with the information is not completely clear grey system, and the accuracy of the evaluation problem of the small sample is not regular.

\subsection{Grey correlation degree}

The grey correlation degree is mainly determined by describing the similarity degree between the curve of VFR curve in different winding States and the same test point.

Under normal operating conditions of the transformer windings, the VFR curve is the reference sequence $X_{0}=\left\{x_{0}(1), x_{0}(2), \cdots, x_{0}(N)\right\}$, and the loose condition is $X_{i}=\left\{x_{i}(1), x_{i}(2), \cdots, x_{i}(N)\right\}$. Among them, $\mathrm{N}$ is the length of the data sequence, and $i=(1,2, \cdots, m)$ is the number of the sequence to be determined. The gray correlation coefficient can be expressed as:

$$
\begin{gathered}
\xi_{i}(k)=\frac{\min _{i} \min _{k}\left|x_{0}(k)-x_{i}(k)\right|+\rho \max _{i} \max _{k}\left|x_{0}(k)-x_{i}(k)\right|}{\left|x_{0}(k)-x_{i}(k)\right|+\rho \max _{i} \max _{k}\left|x_{0}(k)-x_{i}(k)\right|} \\
\gamma\left(X_{0}, X_{I}\right)=\frac{1}{N} \sum_{k=1}^{N} \xi_{i}(k)
\end{gathered}
$$

In Equation (2) and (3): $\xi(k)$ is the correlation coefficient of the point $\mathrm{K} ; \gamma_{i}$ is the correlation degree; $\rho$ is the resolution factor. In order to effectively reduce the correlation degree error, this paper takes $\rho=0.5[16]$.

\subsection{Grey area correlation degree}

The color area correlation is measured by describing the relative area between the VFR curve of the different winding States and the same test point. The calculation formula for the grey area correlation degree is:

$$
\begin{gathered}
\gamma_{i}=\frac{1}{1+k \sum_{k=1}^{N-1} S_{k}} \\
S_{v}=\frac{1}{n \times m} \sum_{i=1}^{m} \sum_{k=1}^{n}\left|S_{i k}\right|
\end{gathered}
$$

\subsection{Analysis of entropy weight correlation degree}

Gray correlation and area correlation analysis respectively from the discrete degree and changing tendency of the curve reflects the characteristics of frequency response curve, the two complement 
each other. However, the vibration signal of the transformer is influenced by the transmission way, the sensor position and the dispersion of the oil tank structure. According to the change trend of VFR curve at single measuring point, it is difficult to judge the state of transformer winding accurately. It is necessary to analyze the characteristic of VFR curve at all measurement points. Among them, it is the key to give different weights to the degree of importance of the relevant characteristics of each measurement point. As an objective evaluation method, the entropy weight method [18] is based on the actual data, which has the advantage of high precision. Therefore, in this paper, by giving each test point in the VFR curve characteristic index of different entropy weight coefficient to reflect the importance of its vibration information. And then define the entropy weight correlation degree to judge the state of the transformer winding.

The grey correlation degree of the VFR curve at each measurement point and the grey area correlation degree is the $\mathrm{M}$ curve evaluation index, then The evaluation matrix of integrated $\mathrm{n}$ test points is $\mathrm{X}$, as follows:

$$
\boldsymbol{X}=\left(x_{i j}\right)_{m \times n}(i=1,2, \cdots, m ; j=1,2, \cdots, n)
$$

The elements in the matrix A are normalized, as follows:

$$
P_{i j}=x_{i j} / \sum_{i=1}^{m} x_{i j}
$$

In equation (7): Pij is the normalized index data

On the basis of the above, the entropy of the evaluation index and the entropy weight of each test point are respectively $W_{j}$ and $E_{j}$, as follows :

$$
\begin{gathered}
w_{j}=\frac{1-E_{j}}{\sum_{j=1}^{n}\left(1-E_{j}\right)} \\
E_{j}=\frac{\sum_{i=1}^{m} P_{i j} \ln P_{i j}}{\ln m}
\end{gathered}
$$

In equation (8) and (9): $E j$ is the entropy of evaluation index; $P i j$ is the standard index data.

Finally, the index of entropy weight correlation is defined to determine the state of the winding, as follows:

$$
\eta=\frac{1}{2}\left[\sum_{k=1}^{N} w_{k} \gamma_{i}(k)+\sum_{l=1}^{N} w_{l} \gamma_{j}(l)\right]
$$

In equation (10): $\gamma_{i}$ and $\gamma_{j}$ are respectively the grey correlation degree and the area correlation degree of VFR curve at each measurement point. $w$ is the entropy weight coefficient, the larger the coefficient value indicates that the impact of the index on the results is greater.

\section{Result analysis}

\subsection{Repeated test results}

Under the normal working condition of the transformer winding two times of repeated tests, the calculation results of the grey correlation degree and the area correlation degree of the VFR curve at 
each measurement point are shown in Table 2. From the table shows that the grey correlation degree and the area correlation degree of the VFR curve at each measuring point are all over 0.9 , and the repeatability of the test of the transformer winding vibration frequency sweep is verified again.

Table 2. Calculated results of correlation of VFR curves

\begin{tabular}{|c|c|c|}
\hline Test point & Grey correlation degree & Area correlation degree \\
\hline 1 & 0.9781 & 0.9635 \\
\hline 2 & 0.9141 & 0.9021 \\
\hline 3 & 0.9798 & 0.9134 \\
\hline 4 & 0.9645 & 0.9698 \\
\hline 5 & 0.9134 & 0.8923 \\
\hline 6 & 0.9256 & 0.9003 \\
\hline 7 & 0.9507 & 0.9125 \\
\hline 8 & 0.9224 & 0.8992 \\
\hline 9 & 0.9318 & 0.9124 \\
\hline 10 & 0.9568 & 0.9453 \\
\hline 11 & 0.9436 & 0.9031 \\
\hline
\end{tabular}

\subsection{The result of correlation degree of entropy weight of transformer winding}

Table 3. Grey correlation results of VFR curves for loosened transformer winding

\begin{tabular}{|c|c|c|c|}
\hline Test point & Loose 1 & Loose 2 & Loose 3 \\
\hline 1 & 0.8862 & 0.7625 & 0.6084 \\
\hline 2 & 0.8800 & 0.7671 & 0.5346 \\
\hline 3 & 0.9237 & 0.8171 & 0.6065 \\
\hline 4 & 0.8222 & 0.7102 & 0.5083 \\
\hline 5 & 0.7928 & 0.6646 & 0.6816 \\
\hline 6 & 0.8327 & 0.7314 & 0.6135 \\
\hline 7 & 0.8117 & 0.7010 & 0.5984 \\
\hline 8 & 0.7717 & 0.6634 & 0.6862 \\
\hline 9 & 0.8242 & 0.6227 & 0.4992 \\
\hline 10 & 0.8347 & 0.6275 & 0.5875 \\
\hline 11 & 0.8152 & 0.6034 & 0.4879 \\
\hline
\end{tabular}

The VFR curve is used as the standard sequence under the first normal operating condition. The grey correlation degree and the area correlation degree of the VFR curve at each measuring point in the normal and loose windings are calculated. The results are shown in Table 3 and 4 respectively. From the table 4 and table 3, we can see that when the transformer winding is loose, the VFR curve of the grey correlation degree and the area correlation degree are decreased. And with the increase of the degree of the transformer winding, the degree of correlation is gradually reduced. It shows that the grey correlation degree and the area correlation degree can be used to measure the variation trend of VFR curve. However, the grey correlation coefficient of VFR curve at the test point 3 is higher, as shown in table 3 . The trends of the grey correlation degree of VFR curve at the point 5 and point 8 and the area correlation degree of the measuring point 5 is not consistent with the preset working condition of the transformer winding. Obviously, by the way of signal transmission and the influence of different measuring points, it is difficult to accurately detect the change of the winding preload according to the change of the VFR curve at the single measuring point. It is necessary to analyze the VFR curves of all measuring points. 
Table 4. Grey area correlation results of VFR curves for loosened transformer winding

\begin{tabular}{|c|c|c|c|}
\hline Test point & Loose 1 & Loose 2 & Loose 3 \\
\hline 1 & 0.8812 & 0.6931 & 0.5253 \\
\hline 2 & 0.6796 & 0.5392 & 0.4005 \\
\hline 3 & 0.7205 & 0.6054 & 0.4782 \\
\hline 4 & 0.7550 & 0.5168 & 0.3868 \\
\hline 5 & 0.8159 & 0.6251 & 0.6980 \\
\hline 6 & 0.6992 & 0.5239 & 0.3699 \\
\hline 7 & 0.7071 & 0.6044 & 0.3997 \\
\hline 8 & 0.7285 & 0.5629 & 0.5343 \\
\hline 9 & 0.6913 & 0.5016 & 0.3532 \\
\hline 10 & 0.6290 & 0.5006 & 0.3831 \\
\hline 11 & 0.7787 & 0.6345 & 0.3988 \\
\hline
\end{tabular}

Table 5. Calculated results of entropy weight coefficients in measured points

\begin{tabular}{|c|c|c|c|c|}
\hline Test point & Normal & Loose 1 & Loose 2 & Loose 3 \\
\hline 1 & 0.0197 & 0.0001 & 0.0156 & 0.0187 \\
\hline 2 & 0.0152 & 0.2204 & 0.1102 & 0.0719 \\
\hline 3 & 0.4276 & 0.0632 & 0.1527 & 0.0488 \\
\hline 4 & 0.0475 & 0.0242 & 0.1714 & 0.0643 \\
\hline 5 & 0.0026 & 0.0027 & 0.0064 & 0.0005 \\
\hline 6 & 0.0667 & 0.1012 & 0.0871 & 0.2159 \\
\hline 7 & 0.1461 & 0.2038 & 0.0462 & 0.1389 \\
\hline 8 & 0.0564 & 0.0110 & 0.0043 & 0.0041 \\
\hline 9 & 0.0385 & 0.1025 & 0.1799 & 0.1526 \\
\hline 10 & 0.1672 & 0.2638 & 0.1886 & 0.1556 \\
\hline 11 & 0.0127 & 0.0070 & 0.0377 & 0.1287 \\
\hline
\end{tabular}

The entropy weight coefficient of each measurement point calculated by the correlation degree of Table 3 and Table 4 is shown in Table 5. According to the equation (10), the calculation results of entropy weight correlation degree can be obtained as shown in table 6 . In Table 6 , the rate of change of the entropy weight is relative to the normal state of the transformer winding. From the Table 6 , the correlation degree of the entropy weight of the VFR curve is decreased with the increase of the loosening degree of the transformer winding. According to the transformer winding looseness preset conditions, when the pressure screw loosening 20\%, entropy correlation decreased $13.49 \%$; when the pressure screw loosening $40 \%$, entropy correlation degree down 33.62\%; when the pressure screw loosening60\%, entropy correlation decreased $48.37 \%$. Therefore, according to the change of the entropy weight of the transformer winding VFR curve, the loosening degree of the winding is detected. When the transformer winding is loose to a certain extent, the weight of the winding itself will play an inhibitory effect on the amplitude of the vibration of the transformer. So that the vibration response of the transformer winding at this time is decreased with the initial loosening of the winding.

Table 6. Calculated results of entropy weight correlation

\begin{tabular}{|c|c|c|c|c|}
\hline & Normal & Loose 1 & Loose 2 & Loose 3 \\
\hline Entropy weight correlation degree & 0.9332 & 0.8073 & 0.6195 & 0.4818 \\
\hline Change rate & - & 13.49 & 33.62 & 48.37 \\
\hline
\end{tabular}




\subsection{The test result of FRA}

Test results of FRA under normal and loose condition of the test transformer winding are shown in figure 6 . The correlation coefficient of low frequency, intermediate frequency and high frequency of FRA curve is calculated according to the standard of DLT911-2004 electric power industry. Among them: low frequency, $1 \sim 100 \mathrm{kHz}$; intermediate frequency, $100 \sim 600 \mathrm{kHz}$; high frequency, $600 \sim 1000 \mathrm{kHz}$. The calculation results are shown in table 7. In figure 7 and table 6 , according to the criteria of FRA method, the different winding loose state of the test transformer, FRA analysis results are normal. Obviously, this is not consistent with the state of the transformer winding in the test. The reason may be due to the distribution parameters of transformer windings, such as inductance, capacitance and so on are not sensitive to the winding. However, the vibration response of the transformer winding is closely related to its mechanical properties. So the method of vibration frequency response can sensitively reflect the loose state of the transformer winding.

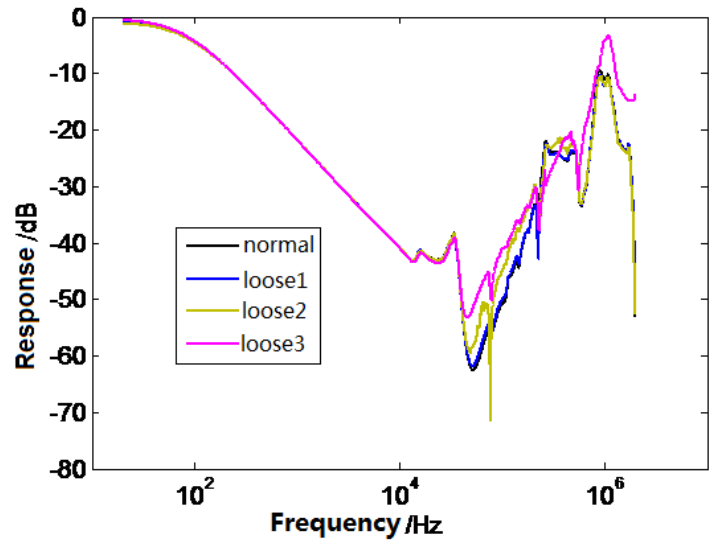

Figure 6. FRA curves of transformer windings under normal and loosened conditions

Table 7. Correlation coefficient of FRA curves

\begin{tabular}{|c|c|c|c|}
\hline & Loose 1 & Loose 2 & Loose \\
\hline Low frequency & 3.0470 & 2.1034 & 2.0511 \\
\hline intermediate frequency & 1.8737 & 1.7564 & 1.1004 \\
\hline high frequency & 2.8796 & 3.0470 & 1.5622 \\
\hline
\end{tabular}

\section{Conclusion}

Based on the physical transformer winding vibration frequency response test results, by introducing the grey relational theory and entropy theory, this paper puts forward the transformer winding vibration frequency detection method to detect the state of windings based on entropy correlation. The following conclusions can be obtained:

(1) Compared with the FRA method, the method of Vibration Frequency Response can be more sensitive to reflect the loose state of the transformer winding. The definition of entropy weight correlation degree can be more accurate detection of the degree of the degree of the winding, which can be found in a timely manner to avoid the fault of the transformer winding, to avoid the occurrence of major accidents.

(2) The grey correlation theory and entropy weight theory can accurately describe the quantitative indexes and the correlation of the VFR curve at each measuring point of the transformer. And it has improved the accuracy of the transformer winding state detection results.

(3) The frequency sweep test of transformer winding has good repeatability. And the connection mode is simple and convenient, and can be used as an important supplement for the routine test of the transformer. 
However, the vibration response of the transformer winding is closely related to its structural characteristics and the position of the measuring points. According to the definition of entropy weight correlation degree of the general application of the transformer winding condition detection still need a lot of testing work, this is our next step of work.

\section{References}

1. JIN Wenlong, CHEN Jianhua, LI Guangfan, et al. Statistics and analysis on power transformer damages caused by short circuit fault in $110 \mathrm{kV}$ and higher voltage classes[J]. Power System Technology, 23(6): 70-74(1999).

2. LUO Bo, WANG Fenghua, JIN Zhijian. A tank vibration model for online monitoring of power transformer[C]// Power and Energy Society General Meeting (PES), 2013 IEEE. IEEE, 2013: 1-5.

3. PATEL M R. Dynamic Response of Power Transformers Under Axial Short Circuit Forces Part IWinding and Clamp as Individual Components[J]. Power Apparatus and Systems, IEEE Transactions on, 1973 (5): 1558-1566.

4. HONG Kaixing, PAN Zaiping. Vibration model of power transformer under short-circuit condition[C]// Electrical Machines and Systems, 2009. ICEMS 2009. International Conference on. IEEE, 1-5(2009).

5. JI Shengchang, LIU Weiguo, SHAN Ping, et al. The application of the wavelet packet to the monitoring of the core and winding condition of transformer[J]. Proceedings of the CSEE, 21(12): 24-27.(2001)

6. XIONG Weihua, ZHAO Guangzhou. Analysis of transformer core vibration characteristics using Hilbert-Huang transformation[J]. 21(8): 9-13 (2006).

7. BORUCKI S. Diagnosis of technical condition of power transformers based on the analysis of vibroacoustic signals measured in transient operating conditions[J]. Power Delivery, IEEE Transactions on, 27(2): 670-676(2012).

8. JI Shengchang, LI Yanming, FU Chenzhao. Application of on-load current method in monitoring the condition of transformer's core based on the vibration analysis method[J]. Proceedings of the CSEE, 23(6): 154-158(2003).

9. GARCIA B, BURGOS J C, ALONSO A M. Transformer tank vibration modeling as a method detecting winding deformations: Part I theoretical foundation[J]. IEEE Trans on Power Delivery,21(1): 157-163(2006).

10. GARCIA B, BURGOS J C, ALONSO A M. Transformer tank vibration modeling as a method detecting winding deformations: Part II experimental verification[J]. IEEE Trans on Power Delivery,21(1): 164-169(2006).

11. LIU Hongtao, MA Hongzhong, GAO Peng, et al. Method of determining the threshold of transformer fault using transformer tank vibration model[J]. Proceedings of the CSU-EPSA, , 24(6): 36-40+82(2012).

12. MA Hongzhong, GENG Zhihui, CHEN Kai, et al. A new fault diagnosis method for power transformer winding deformation based on vibration[J]. Automation of Electric Power System, 37(8): 89-95(2013).

13. XU Jian, SHAO Yuying, WANG Fenghua, et al. Comparative research on behavior of vibration frequency response analysis and frequency response analysis in detection of transformer winding deformation[J]. Power System Technology, 35(6): 213-218(2011).

14. SHAO Yuying, XU Jian, RAO Zhushi, et al. Application research of detecting transformer winding's deformation based on vibration frequency response analysis[J]. JOURNAL OF SHANGHAI JIAO TONG UNIVERSITY, 2010(9): 1223-1228.

15. LI Jianpo, ZHAO Jiyin, ZHENG Ruirui. Power transformer fault diagnosis based on comprehensive correlation analysis[J]. Journal of Jilin University(Information Science Edition),26(1): 62-68(2008).

16. LV Ganyun, CHENG Haozhong, ZHAI Haibao, et al. Fault diagnosis of power transformer based on improved grey relation analysis[J]. Proceedings of CSEE, 24(10): 123-128(2004). 
17. SUN Caixin, LI Jian, ZHENG Haiping, et al. A new method of faulty insulation diagnosis in power transformer based on degree of area incidence analysis[J]. Power System Technology. 26(7): 24-29(2002).

18. CHENG Qiyue. Structure entropy weight method to confirm the weight of evaluating index[J]. Systems Engineering Theory \& Practice. 30(7): 1225-1228 (2010)

19. DL/T 911-2004 Frequency response analysis on winding deformation of power transformers[S]. Beijing: China Electric Power Press,(2004). 